\title{
Microbiology of chronic suppurative otitis media at Queen Elizabeth Central Hospital, Blantyre, Malawi: A cross-sectional descriptive study
}

\section{Chirwa ${ }^{1}$,W Mulwafu ${ }^{2}$, J MAswani ${ }^{3}$, PW Masinde ${ }^{3}$, R Mkakosya ${ }^{4}$, D Soko ${ }^{4}$}

1.ENT Department, Kamuzu Central Hospital, Lilongwe, Malawi 2.College of Medicine, Univeristy of Malawi and Queen Elizabeth Central Hospital, Blantyre, Malawi

3.Department of ENT/Head and Neck Surgery, University of Nairobi, Kenya

4.Department of Pathology and Laboratory Sciences, College of Medicine, Blantyre Malawi

\section{Abstract}

\section{Background}

Chronic suppurative otitis media (CSOM) is still a significant health problem in developing countries. Therefore, it was pertinent to determine the local Malawian microbiology in order to guide adequate treatment, avoid complications, and provide records for future reference.

\section{Aim}

The study sought to determine the CSOM-causing microorganisms at Queen Elizabeth Central Hospital in Blantyre, Malawi, and establish their relationship signs and symptoms, and with the demographic pattern of the study.

\section{Methods}

This was a hospital-based cross-sectional descriptive study carried out at the ENT outpatient clinic and the Microbiology Department of Queen Elizabeth Central Hospital.The sample comprised 104 patients with unilateral or bilateral active CSOM, who met the inclusion criteria. All patients were evaluated through a detailed history and clinical examination. Pus samples from draining ears were collected by aspiration with a sterile pipette. The specimens were immediately sent for microbiological analysis. Data were analyzed using SPSS.version 20

\section{Results}

The study found that Proteus mirabilis, Pseudomonas aeruginosa, and Staphylococcus aureus were the most prevalent aerobic bacteria, while Bacteroides spp. and Peptostreptococcus spp. were the commonest anaerobic bacteria causing CSOM. These CSOM-causing microorganisms were predominant among males aged 18 years and below. Some CSOMcausing microorganisms were-significantly more so than the otherscharacteristically associated with each of the following clinical features: quantity of pus drainage, mode of onset, otalgia, hearing loss, location of tympanic membrane perforation, and mucosal appearance.

\section{Introduction}

Chronic suppurative otitis media (CSOM) is defined as a perforation of the tympanic membrane, with persistent drainage of pus from the middle ear, lasting at least two weeks ${ }^{1}$. The global burden of illness from CSOM is estimated to involve about 65 to 330 million individuals with draining ears, $60 \%$ (39 to 200 million) of whom suffer from significant hearing impairment.2 Over $90 \%$ of the burden is borne by developing countries in Southeast Asia, the Western Pacific Region, and Africa ${ }^{2}$.

Typical pathogens reach the middle ear following insufflations of respiratory pathogens through the eustachian tubes from the nasopharynx and spread from the external ear canal inwards through a non-intact tympanic membrane ${ }^{3,4}$. Studies on microbiologic diagnoses of CSOM differ in regard to patient age, geography, and the presence of complications such as cholesteatomas, and these inconsistencies likely impact some of the variation in reported pathogens. A portion of the variability observed may be related to differences in sampling and processing methods ${ }^{3,5}$. Knowledge of the true frequency of polymicrobial infection, particularly the extent of anaerobic involvement, is limited by differences in collection and culture techniques ${ }^{6,7}$. Traditional swab specimen collection has been associated with contamination with normal skin flora like Staphylococcus epidermidis, diphtheroids and anaerobic organisms, such as Propionibacterium acnes ${ }^{8}$.

Aerobes, anaerobes, and fungi are all potential pathogens in CSOM. Understanding of the microbiology of chronic otitis media is important for efficient and effective treatment, and prevention of complications and antibiotic resistance. The objective of this study was to determine the CSOM-causing microorganisms and associated factors among CSOM patients attending the ENT clinic at Queen Elizabeth Central Hospital (QECH) in Blantyre, Malawi.

\section{Methods}

This was a hospital-based cross-sectional descriptive study.

This study was carried out in the ENT outpatient clinic at QECH. Queen Elizabeth Central Hosptial has bed capacity of 1,200 beds and serves the population of Blantyre District and the Southern Region of Malawi, which has a population of about 5.5 million people. Microbiological analyses were carried out at the QECH microbiology laboratory and University of Malawi College of Medicine's laboratory.

The study was done between July and September 2013. The study population comprised CSOM patients attending the ENT outpatient clinic at QECH during the study period. Inclusion criteria were all patients with actively draining CSOM who consented or whose guardians consented to participate in the study and did not meet any of the exclusion criteria. Exclusion criteria were patients on antibiotic or antifungal treatment (ear drops or systemic) within the previous two weeks, patients with draining ears of less than two weeks duration, patients with draining ears but intact tympanic membrane (otitis externa), and patients who refused to consent to participate in the study.

\section{Sample size determination}

The sample size was determined using the Cochran (1963:75) formula to yield a representative sample for proportions. Thus, a sample size of 104 CSOM patients was taken to increase the representativeness of the sample, minimize sampling errors, increase generalizability of the results, and cater for attrition (10\%).

\section{Sampling method}

The study utilized simple random sampling to select CSOM patients. Subjects were recruited into the study as they came to the clinic until the required number was obtained with strict application of the inclusion and exclusion criteria.

\section{Social demographic and medical history}

A detailed clinical history that captured age, gender, duration of discharge and antibiotic therapy was taken for all included http://dx.doi.org/10.4314/mmj.v27i4.1 
patients. Patients of any age, both genders, unilateral or bilateral draining ears, resulting from CSOM of more than two weeks, were included in the study. Demographic data were taken and, together with the patient's medical history and physical examination findings, were entered in the patient's study file.

\section{Bacterial isolation}

Pus specimens from draining ears were taken on the first day of contact with the patient. The ears were inspected first; pus from the outer part of the ear canal was then cleaned by suction. A sterile pipette was then introduced through a sterile aural speculum placed in the external auditory canal, and each specimen was aspirated from the bony part of the ear canal (inner two- thirds) or the middle ear cavity. Pus specimens were collected from both ears for patients with bilateral draining. Each collected specimen was immediately placed in an anaerobic jar, under aseptic conditions, and transported, within one hour of collection, to the microbiology laboratory for routine microbiological culture and identification.

For bacterial isolation, the specimens were inoculated on blood agar, MacConkey's agar, chocolate agar and Robertson's cooked meat medium for aerobic and anaerobic cultures. For anaerobic bacteria, anaerobic blood agars were incubated in an anaerobic jar to permit recovery of anaerobic pathogens. For fungi isolation, a part of the pus specimen was cultured on Sabouraud's dextrose agar. The culture plates were incubated at $37^{\circ} \mathrm{C}$ for 24 to 48 hours. Anaerobic culture plates were incubated for seven days to allow growth of anaerobes, which grow slowly compared to aerobes. The isolates from the culture plates were identified using Gram staining, colony morphology, catalase, coagulase, oxidase, and biochemical strips. For fungal growth, lactophenol cotton blue was used for final identification, and culture was for no less than seven days.

\section{Data management}

All data collected in the study were sorted, coded, and entered in a computer using SPSS version 20 software. Data were cross-checked against the data files for any inconsistencies or obvious data entry errors. The laboratory request form was also checked for the desired test. The data entry and editing was done throughout the study process. The demographic details, characteristics, and particulars of the subjects, in terms of predictability and determination of risk of CSOM, were analyzed using the Chi-square test. Cross tabulations were done to establish relationships between variables and Chi-square tests were used to test association. Data from bacterial isolation were analyzed using qualitative methods.

\section{Ethical consideration}

Ethical approval was obtained from the University of Malawi's College of Medicine Research and Ethics Committee.

\section{Results}

There were 64 males $(61.5 \%)$ and 40 females (38.5\%). The mean age was 17.8 years where $64(61.5 \%)$ were aged below 18 years and $40(38.5 \%)$ were aged 18 years and above. The range of the ages was 2 to 64 years, and the median age was 14 years.

\section{Clinical presentation}

There were 90 patients $(86.5 \%)$ with unilateral disease and 14 $(6.7 \%)$ with bilateral disease. The commonest mode of onset was ear pain in 87 study patients $(73.8 \%)$, followed by upper respiratory tract infection (URTI) in 19 (16.1\%), trauma in $7(5.9 \%)$, and foreign body in 5 patients (4.2\%). Hearing loss was reported in 100 ears $(84.7 \%)$; it was persistent in 69 ears (68.6\%) and fluctuating in 31 (31.4\%). Otorrhoea was observed in the left ear in 61 patients $(51.7 \%)$ and in the right ear in 57 (48.3\%). Pus drainage was foul smelling in 90 ears $(76.2 \%)$ and odourless in $28(23.8 \%)$. The pattern of drainage was continuous in 65 ears $(55.1 \%)$ and recurrent in $53(44.9 \%)$ (Figure 1).

\section{Figure 1: Type and pattern of pus drainage}

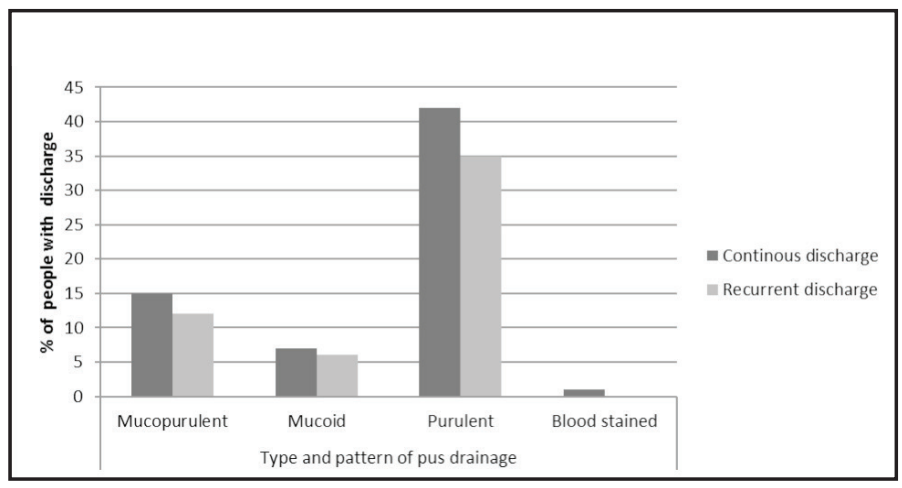

Examination findings showed that the quantity of pus in the canal was scanty in 63 ears (53.4\%) and copius in the other 55 (46.6\%). The location of tympanic membrane perforation was central in 54 ears $(45.8 \%)$, subtotal in 27 $(22.9 \%)$, marginal in $26(22.0 \%)$, attic in $9(7.6 \%)$, and total perforation in 2 ears $(1.7 \%)$. Mucosal appearance in the middle ear was injected in $57(48.4 \%)$, hyperplastic in 41 $(34.7 \%)$, and sclerotic in 20 ears $(16.9 \%)$. Granulation tissue was present in 20 ears $(16.9 \%)$.

\section{Laboratory findings}

There was an equal number $(\mathrm{n}=58 ; 49.2 \%)$ of mixed and pure cultures, while $2(1.7 \%)$ specimens produced no growth. On Gram staining, gram-negative bacteria accounted for 84 specimens $(72.4 \%)$ and gram-positive bacteria were found in 32 specimens (27.6\%). Bacterial findings were categorized into aerobes and anaerobes. The most common aerobes identified from the specimens were gram-negative bacteria, which included Proteus mirabilis (n $=44 ; 28.6 \%$ ), Pseudomonas aeruginosa ( $\mathrm{n}=32 ; 20.8 \%$ ), and Escherichia coli ( $\mathrm{n}=13 ; 8.4 \%$ ). The gram-positive aerobes identified included Staphylococcus aureus $(\mathrm{n}=31$; $20.1 \%$ ) and coagulase-negative staphylococci $(n=6 ; 4.0 \%)$. Other organisms were identified in small numbers (Table 1). Anaerobes were isolated from 39 (33.6\%) of the specimens. The most common anaerobes identified were Bacteroides spp. in 18 specimens (15.5\%), Peptostreptococcus spp. in $12(10.3 \%)$, and Clostridium spp. in 7 (6.0\%) (Table 2). Half of the positive cultures were mixed cultures. Aerobes were identified in the mixed cultures, and mixtures of $\mathrm{P}$. mirabilis and $\mathrm{P}$. aeruginosa were the most common finding ( $\mathrm{n}=19 ; 16.4 \%$ ). Mixtures of P. mirabilis and E. coli were found in 8 specimens $(6.9 \%)$ and S. aureus with P. mirabilis was found in 7 specimens $(6.0 \%)$. Mixtures of aerobes and anaerobes were isolated from $34(29.3 \%)$ of the cultures. These comprised mainly Bacteroides spp. ( $n=15 ; 12.9 \%$ ), Peptostreptococcus spp. ( $\mathrm{n}=9 ; 7.8 \%$ ) and Clostridium spp. ( $\mathrm{n}=6 ; 5.2 \%$ ). Others were observed in small numbers.

Fungal isolates were identified in 21 specimens (18.1\%). The fungal isolates consisted of Aspergillus spp. $(\mathrm{n}=12 ; 10.3 \%)$ and Candida spp. $(\mathrm{n}=9 ; 7.8 \%)$. 
Tablel: Aerobes isolated

\begin{tabular}{lll|}
\hline Species & & \\
\hline Gram-positive aerobes & Frequency & Percent \\
Staphylococcus aureus & 31 & $20.1 \%$ \\
Coagulase-negative staphylococci & 6 & $4.0 \%$ \\
Streptococcus pyogenes & 3 & $1.9 \%$ \\
Streptococcus pneumoniae & 2 & $1.3 \%$ \\
\hline Total & $\mathbf{4 2}$ & $\mathbf{2 7 . 3 \%}$ \\
\hline Gram-negative aerobes & & \\
Proteus mirabilis & 44 & $28.6 \%$ \\
Pseudomonas aeruginosa & 32 & $20.8 \%$ \\
Escherichia coli & 13 & $8.4 \%$ \\
Klebsiella pneumoniae & 6 & $4.0 \%$ \\
Proteus vulgaris & 6 & $4.0 \%$ \\
Diptheroides spp. & 4 & $2.6 \%$ \\
Enterobacter cloacae & 3 & $1.9 \%$ \\
Morganella morganii & 1 & $0.6 \%$ \\
Raultella ornithinolytica & 1 & $0.6 \%$ \\
Coliforms & 1 & $0.6 \%$ \\
Enterobacter cloacae & 1 & $0.6 \%$ \\
\hline Total & $\mathbf{1 1 2}$ & $\mathbf{7 2 . 7 \%}$ \\
\hline
\end{tabular}

Table 2: Anaerobes isolated

\begin{tabular}{|lll|}
\hline Species & Frequency & Percent \\
\hline Bacteroides spp. & 18 & $15.6 \%$ \\
Peptostreptococcus spp. & 12 & $10.3 \%$ \\
Clostridium spp. & 7 & $6.0 \%$ \\
Prevotella melaninogenica & 2 & $1.7 \%$ \\
\hline Total & $\mathbf{3 9}$ & $\mathbf{3 3 . 6 \%}$ \\
\hline & \\
\hline
\end{tabular}

Table 3: Associations between CSOM-causing microorganisms and clinical features

\begin{tabular}{|lccr|}
\hline Clinical feature & Wald chi-square & df & p-value \\
\hline Otorrhoea & 0.046 & 2 & 0.831 \\
Type of discharge & 1.027 & 2 & 0.598 \\
Quantity of discharge & 12.208 & 2 & $\mathbf{0 . 0 0 2}$ \\
Type of odour & 0.682 & 1 & 0.409 \\
Mode of onset & 13.565 & 4 & $\mathbf{0 . 0 0 9}$ \\
Presence of otalgia & 5.054 & 1 & $\mathbf{0 . 0 2 5}$ \\
Presence of hearing loss & 10.628 & 1 & $\mathbf{0 . 0 0 1}$ \\
Character of pus discharged & 8.518 & 4 & 0.074 \\
Location of TM perforation & 38.279 & 3 & $\mathbf{0 . 0 0 0}$ \\
Mucosal appearance & 35.445 & 2 & $\mathbf{0 . 0 0 0}$ \\
Granulation tissue & 0.478 & 1 & 0.489 \\
\hline TM tympanic membrane & & & \\
& & & \\
\hline
\end{tabular}

Table 4: Most common organisms associated with specific clinical features of CSOM

\begin{tabular}{|ll|}
\hline Clinical feature & Most common organism \\
\hline Continuous pus drainage & P. mirabilis \\
Recurrent pus discharge & S. aureus \\
Otalgia & P. mirabilis \\
Persistent hearing loss & S. aureus \\
Central perforation & P. mirabilis \\
Marginal perforation & Aspergillus spp. \\
\hline
\end{tabular}

\section{Association between CSOM-causing microorganisms and symptoms and signs}

The chi-square test was used to determine the relationship between the different CSOM-causing microorganisms and symptoms and signs. Some CSOM-causing microorganisms were-significantly more so than the others $(\mathrm{p}<0.05)$ characteristically associated with each of the following clinical features: quantity of pus drainage, mode of onset, otalgia, hearing loss, location of tympanic membrane perforation and mucosal appearance (Table 3, Table 4).

Continuous pus drainage was associated with 20 P. mirabilis cultures $(17.2 \%), 11$ P. aeruginosa cultures $(9.5 \%), 8$ Bacteroides cultures, and 8 Aspergillus cultures (6.9\%). S. aureus was mostly associated with recurrent discharge (6 specimens; $5.2 \%$ ). The mode of onset was mainly acute ear pain in specimens with P. mirabilis ( $\mathrm{n}=16 ; 13.7 \%)$, P. aeruginosa $(n=15 ; 12.8 \%)$, and Aspergillus spp. $(n=9 ; 7.7 \%)$. Otalgia was most commonly associated with P. mirabilis (n $=26 ; 22.0 \%)$, P. aeruginosa $(\mathrm{n}=19 ; 16.1 \%)$, S. aureus ( $\mathrm{n}$ $=13 ; 11.0 \%)$, and Aspergillus spp. $(\mathrm{n}=14 ; \mathrm{p}=11.9 \%)$. Persistent hearing loss was mainly present in ears whose specimens grew $\mathrm{S}$. aureus $(\mathrm{n}=16 ; 16.0 \%)$, P. aeruginosa $(\mathrm{n}=$ 13; 13.0\%), Bacteroides spp. $(\mathrm{n}=7 ; 7.0 \%)$, and Aspergillus spp. $(\mathrm{n}=7 ; 7.0 \%)$. The location of tympanic membrane perforation was mainly central in ears whose specimens grew P. mirabilis ( $\mathrm{n}=15 ; 12.7 \%)$, P. aeruginosa $(\mathrm{n}=9 ; 7.6 \%)$ and Aspergillus spp. $(\mathrm{n}=6 ; 5.1 \%)$. Subtotal tympanic membrane perforations were mainly associated with specimens that grew $S$. aureus $(n=5 ; 4.2 \%)$ and marginal perforations were associated mostly with specimens that grew Aspergillus spp. $(\mathrm{n}=12 ; 12.0 \%)$ and P. mirabilis $(\mathrm{n}=7 ; 5.9 \%)$. The mucosal appearance was mainly injected in ears whose specimens grew S. aureus $(\mathrm{n}=6 ; 5.2 \%$ ) and Candida spp. ( $\mathrm{n}=5 ; 4.2 \%$ ). Hyperplastic mucosal appearance was mostly associated with P. mirabilis ( $\mathrm{n}=11 ; 9.3 \%)$, P. aeruginosa $(\mathrm{n}=6 ; 5.1 \%)$, and Candida spp. ( $\mathrm{n}=3 ; 2.5 \%)$. Sclerotic mucosa was mostly associated with specimens that grew coliforms, P. vulgaris and Peptostreptococcus spp.(Table 4).

\section{Discussion}

CSOM was most prevalent in children and young adults than in older participants. This is similar to studies reported by others in India and Pakistan ${ }^{9,10}$. There are several reasons to explain this observation. The eustachian tubes in children are shorter, narrower, and more horizontal than in adults, and frequent upper respiratory tract infections are more common in children ${ }^{11}$. However, these findings differ from the findings of another study from Singapore, which showed that the disease was more prevalent in the age group of 31 to 40 years $^{12}$. In our study, males were more affected than females, and this is similar to findings reported from Ethiopia ${ }^{13}$.

Otorrhoea was observed in the left ear in 61 cases $(51.7 \%)$, and in the right ear in 57 cases (48.3\%). There were 7 bilateral cases. This is comparable with results from a local study and a study from Nigeria ${ }^{14}$. Patients in our study, who had bilateral disease, exhibited no differences in terms of CSOM-causing microorganisms between the right and left ears. However, in one study conducted in South Africa, $44.4 \%$ of patients with bilateral disease showed differences in the distribution of bacteria, indicating that separate pus specimens need to be taken in bilateral disease ${ }^{16}$. The commonest mode 
of onset was acute ear pain, which was reported by nearly three-quarters of the patients. Otalgia was present in 111 ears $(94.1 \%)$, which was higher compared to a study in Iraq, where the prevalence of otalgia was $41.7 \%{ }^{17}$.

The pus drainage was mainly purulent and foul smelling in 90 ears $(76.2 \%)$ and mucopurulent and odourless in 28 ears $(23.8 \%)$. This is similar to a study in Bangladesh, where aural drainage was mucoid or mucopurulent in $80(80 \%)$ of patients with tubotympanic CSOM and foul smelling, scanty ear drainage was present in $88(88 \%)$ of patients with atticoantral $\mathrm{CSOM}^{18}$. Findings in a study done in Iraq indicated that pus drainage was foul smelling in 30 ears $(25 \%)$, which was different from this study. The difference can be attributed to variation in population characteristics and geographical location.

Hearing loss was persistent in 69 ears (68.6\%) and fluctuating in 31 ears $(31.4 \%)$. This is in agreement with a study by Sheahan et al., where $80 \%$ of cases had hearing loss ${ }^{19}$.

\section{Microbiology}

In the present study, gram-negative rods were more than twice as common as gram-positive. It is usually seen that aerobic gram-negative rods outnumber gram-positive cocci in $\mathrm{CSOM}^{20,21}$. This could be attributed to exposure to contaminated water.

The most common bacterial isolates causing CSOM in this study were P. mirabilis, P. aeruginosa, and S. aureus. A study conducted in rural Malawi in 1998 also showed that P. mirabilis was the commonest aerobic bacteria ${ }^{22}$. The findings from the rural setting were similar to those in this study from an urban setting of Malawi in terms of CSOMcausing microorganisms. P. mirabilis has been found to be the commonest isolate in rural Kenya, ${ }^{23}$ as well as in urban areas of Congo $^{24}$ and Ethiopia ${ }^{13}$. Findings of P. mirabilis as a common isolate suggests that infections are from the community, where there is a constant circulation of Proteus and Staphylococcus species, as opposed to the health facilities. Although the recommended treatment for Pseudomonas aeruginosa is ciprofloxacin ear drops, this is not available in most health facilities in Malawi. As such, treatment with ciprofloxacin ear drops may not explain the relatively lower yield of $\mathrm{P}$. aeruginosa ( $\mathrm{n}=32 ; 20.8 \%$ ), compared to other studies $^{25,26,27}$. Various researchers, over the past few decades, have isolated P. aeruginosa from 48 to $98 \%$ of patients with CSOM. Other studies have shown that $\mathrm{S}$. aureus is the most common, especially when cholesteatoma is present ${ }^{25,26,27}$. The ability of these organisms to form biofilms may contribute to their frequency in CSOM. In this study, E.coli was isolated from 13 cultures (8.4\%) and Klebsiella spp. from $6(3.9 \%)$; these are comparable to findings reported elsewhere.22,10 More frequent isolation of faecal bacteria, like E.coli and Klebsiella spp., indicates that individuals are at high risk of infection from poor hygiene and sanitary conditions.

In this study anaerobes were isolated in $33.1 \%$ of the total sampled specimens, most commonly Bacteroides spp., followed by Peptostreptococcus spp. and Clostridium spp. This is comparable to findings from other reported studies ${ }^{28,16}$. Other research findings have showed Peptococcus spp., Peptostreptococcus spp., and Prevotella melaninogenicus as the commonest anaerobes ${ }^{29,30}$. Some studies have reported isolation rates of anaerobes of $20 \%$ to $50 \% 0^{13,24}$. Improved isolation of anaerobes can be attributed to improved collection, transportation, and processing techniques of the specimens. Mixed aerobic and anaerobic cultures also characterize chronic infection in CSOM, suggesting a potential synergy between aerobic and anaerobic bacteria. It has been reported that polymicrobial infections are more pathogenic than monomicrobial infections ${ }^{31}$.

In this study, fungal isolates were identified in $21(17.8 \%)$ of the sampled specimens, and Aspergillus spp. were the commonest, followed by Candida spp.; this is comparable with results published elsewhere ${ }^{32}$. Fungal infections of the middle ear are common as fungi thrive well in moist ears ${ }^{33}$. The most commonly isolated fungi in CSOM are Candida and Aspergillus ${ }^{34}$.

This study showed statistically significant characteristic associations of some CSOM-causing microorganisms with specific aspects of the CSOM symptomatology, which is contrary to a study where bacteriological findings had no significant effect on symptoms and signs ${ }^{35}$.

One limitation for this study was that antibiotic susceptibility testing was not done. We recommend that future studies evaluate the drug sensitivities of local microorganisms that cause CSOM and subsequently identify changes in bacteriological profile and, possibly, the emergence of multidrug-resistant strains resulting from indiscriminate use of antibacterial agents.

\section{Conclusions}

Proteus mirabilis, Pseudomonas aeruginosa, and Staphylococcus aureus were the most prevalent aerobic bacteria, while Bacteroides spp. and Peptostreptococcus spp. were the commonest CSOM-causing anaerobic bacteria. These CSOM-causing microorganisms were predominant among males aged 18 years and below. The disparity in findings from previous research can be attributed to the variation in climate, community enivronment, patient population, and indiscriminate use of antibiotics. There was a correlation between particular CSOM-causing microorganisms and specific clinical features of CSOM.

\section{References}

1. Smith AW, Macharia I, Mugwe P, Hatcher J. Randomized control trial of treatment of chronic otitis media in Kenyan school Children. Lancet, 1996, 348: 1128-1133

2. Jose A. Chronic otitis media: Burden of Illness and Management .Child and Adolescent Health and Development Prevention of Blindness and Deafness. World Health Organization (WHO). Geneva, Switzerland, 2004.

3. Roland PS. Chronic otitis media: a clinical overview. Ear Nose Throat J 2002; 81: 8

4. Verhoeff $\mathrm{M}$, et al. Chronic otitis media: a review. Int $\mathrm{J}$ Pediatr Otorhinol 2006; 70-71

5. Vartiainen E, Vartiainen J. Effect of aerobic bacteriology on the clinical presentation and treatment results of CSOM. J Laryngol Otol 1996; 110: 315

6. Brook I, Burke P. The management of acute, serous and CSOM: the role of anaerobic bacteria. J Hosp Infect 1992; 22 Suppl A: 75

7. Brook I. The role of anaerobic bacteria in otitis media: microbiology, pathogens-is, and implications on therapy. Am J Otolaryngol 1987; 8: 109

8. Adoga AS, Malu D, Badung BP, Obiesie IV. Swab and Aspiration collection methods and antibiograms in chronic otitis media at Jos University Teaching Hospital. Which is superior? Ann Afr Med 2010 vol 9. Issue 4:230-234 
9. Poorley VK and Lyer A. Study of bacterial flora in chronic otitis media and itsclinical significance. Indian J Otolaryngol Head and Neck Surg 2002;54:91-5

10. Mansoor T, Mussani MA, Khalid G, Kumal M.Pseudomonas aeruginosa in Chronic Otitis media: sensitivity spectrum against various antibiotics in Karachi. J Ayub Med Coll Abbottabad 2009; 21(2):120-23

11. Mawson S, Pollack M. Special role of Pseudomonas aeruginosa in chronic otitis media. Ann Otol Rhinol Laryngol Head and Neck Surg., 1988, 97 (Suppl 130): 10-13 46: 488-97

12. Loy AHC, Tan AL, Lu PKS. Microbiology of chronic otitis media in Singapore. Singapore Med J 2002;43:296-9

13. Melaku A, Lulseged S. Chronic otitis media in a childrens hospital in Addis Ababa, Ethiopia. Ethiop Med J. 1999 Oct; (4): 237-46

14. Van Hasselt P, van Kregten E. Treatment of chronic otitits media with ofloxacin ear drops: A clinical/bacteriological study in a rural area of Malawi. Int J Paedtr Otorhino 2002;63(1):49-56

15. Olatoke F, Ologe FE, Nwawolo CC, Saka MJ. The prevalence of hearing loss among school children with chronic otitis media in Nigeria and its effect on academic performance. Ear Nose Throat $\mathbf{J}$ 2008;87(12):E19

16. Tiedt NJ, Butler IR, Atkins MD, Elliot E et al. Paediatric chronic otitis media in the Free State Province: Clinical and audiological features. The South African Med J; vol103, no 7(2013

17. Ahmed, M., Ihsan E., Jassim M., Prevalence and patterns of chronic suppurative otitis media and hearing impairment in Basrah city Journal of Medicine and Medical Sciences Vol. 1(4) pp. 129-133 May 2010

18. Chowdhury M. A., Alauddin M., comparative study between tubotympanic and atticoantral types of chronic suppurative otitis media Bangladesh Med Res Counc Bull 2002 Apr;28(1):36-44

19. Sheahan P., Donnelly M., Kane R., Clinical features of newly presented cases of CSOM Journal of Laryngology and Otology 2001;115:962-6

20. Kumar H, Seth S. Bacterial and Fungal study of 100 cases of Chronic otitis media. J Clin Diag Res.2011;5:1224-7

21. Shyamla R, Reddy SP. The study of bacteriological agents of chronic otitis media-aerobic culture and evaluation. J Microbiol Biotechnol Res. 2012;(2):152-62

22. Van Hasselt et al. Bacteriology of Chronic Otitis Media amongst children in Nkhotakota District of Malawi ENT and Audiology News Vol. 12 May/June 2013
23. Macharia I, Mugwe P et al. Bacteriology of chronic otitis meida in Garissa district, Kenya: A point prevalence study. International Journal of Paediatric Otorhinolaryngology vol 77, issue 7, July 2013, p11071111

24. Nyembue DT, Tshiswaka JM, Sabue MJ. Bacteriology of Chronic otitis media among Congolese children. Acta Otolaryngol Belg. 2003; 57(3):205-8

25. David S. Haynes. Peril-operative antibiotics in chronic otitis media. Ear, Nose and Throat Journal, August, 2002

26. Constanble L, Butler I. Microbial flora in chronic otitis media. J Infect 1982; 5: 57-60

27. Iquibal SM, Hassan A, Shafiq M. Chronic otitis media: disease pattern and drug sensitivity. J Surg Pak 2006; 11: 17-19

28. Nikakhlagh S, Khosrani A,Mand Rahidi N et al. Microbiological findings in patients with Chronic otitis media. J Med Science. 2008; 8(5): 503-506

29. Sweeney G, Picozzi G, Browning GG. A quantitative study of aerobic and anaerobic bacteria in CSOM. J Infect 1982; 5: 49-55

30. Maji PK, Chatterjee S, Chatterjee J et al. 2007. The investigation of bacteriaology of chronicotitis media in patients attending a tertially care hospital with special emphasis in seasonal variation. Indian J. Otol Head and Neck Surg, 59: 128-131

31. Brook I. Microbiology of polymicrobiol abscesses and implication for therapy. J Antimicrobial chemother 2002;50:805-1

32. Olu Ibekwe A, Zain Al Shareef, Ashraf Benayum. Anaerobes and Fungi in Chronic otitis media. Ann Otol Rhinol Laryngol 106: 1997; 106:694-52

33. Tiwari S et al. Chronic bilateral Suppurative Otitis media caused by Aspergillus terrens, Mycoces 38(7-8)(1995).

34. Rajat P, Deepak J, Vikran N et al. Microbiology of Chronic Otitis media in a Tertially Care Setup of Utterrakhand State. N Am J Med Sci. 2013 April; 5(4):282-285

35. Vartiainen E., Vartiainen J., Effects of aerobic bacteriology on clinical presentation and treatment results of CSOM Journal Laryngology and Otology 1996 April, 110(4):315-8 